\title{
Radar Detection Schemes for Joint Temporal and Spatial Correlated Clutter Using Vector ARMA Models
}

\author{
Wajih Ben Abdallah \\ L2S and SONDRA, CentraleSupélec \\ Gif-sur-Yvette, France
}

\author{
Jean Philippe Ovarlez \\ SONDRA and French Aerospace Lab \\ ONERA DEMR/TSI, Palaiseau, France
}

\author{
Pascal Bondon \\ L2S, CNRS \\ Gif-sur-Yvette, France
}

\begin{abstract}
Adaptive radar detection and estimation schemes are often based on the independence of the training data used for building estimators and detectors. This paper relaxes this constraint and deals with the non-trivial problem of deriving detection and estimation schemes for joint spatial and temporal correlated radar measurements. In order to estimate these two joint correlation matrices, we propose to use the Vector ARMA (VARMA) methodology. The estimation of the VARMA model parameters are performed with Maximum Likelihood Estimators in Gaussian and non-Gaussian environment. These two joint estimates of the spatial and temporal covariance matrices leads to build Adaptive Radar Detectors, like Adaptive Normalized Matched Filter (ANMF). Their corresponding performance are analyzed through simulated datasets. We show that taking into account the spatial covariance matrix may lead to significant performance improvements compared to classical procedures ignoring the spatial correlation.
\end{abstract}

\section{INTRODUCTION}

In many applications, data can be viewed as a joint spatial and temporal process. For example, in meteorology sets of rainfall measurements can be collected from a number of different sites over a period of time [1], [2]. In radar and imagery applications, this view point can be of high interest. For high resolution radar, the sea clutter is clearly jointly spatially and temporally correlated [3]. In multichannel (polarimetric, interferometric or multi-temporal) SAR imaging, the multivariate vector characterizing each spatial pixel of the image is correlated over the channels but can also be strongly correlated with those of neighbourhood pixels. Finally, the need of simulating doubly correlated temporal and spatial process is of importance. In the radar community, one generally supposes that the vectors of information collected over a spatial support are identically and independently distributed (IID). This strong assumption allows to build easily Maximum Likelihood Estimators of parameters like for example, covariance matrix required for adaptive detection, leading to overestimated performance of such detectors. The aim of this paper is to relax this hypothesis through the use of spacetime autoregressive and moving average vector models in the context of wide radar applications.

The vector ARMA (VARMA) model has been widely used for modeling the temporal or spatial dependence structure of a multivariate discrete signal. Unlike scalar signals, the temporal or spatial dependence of a vector signal consists of not only the serial dependence within each marginal signal, but also the interdependence across different marginal signals. The VARMA model is well suited to represent such temporal dependence structures. More precisely, $\left(\boldsymbol{y}_{k}\right)_{k \in \mathbb{Z}}$ is an $m$ variate zero-mean $\operatorname{VARMA}(p, q)$ model if $\left(\boldsymbol{y}_{k}\right)$ satisfies the difference equation

$$
\boldsymbol{y}_{k}-\sum_{i=1}^{p} \boldsymbol{\Phi}_{i} \boldsymbol{y}_{k-i}=\boldsymbol{c}_{k}+\sum_{i=1}^{q} \boldsymbol{\Theta}_{i} \boldsymbol{c}_{k-i}
$$

where $\boldsymbol{\Phi}_{1}, \ldots, \boldsymbol{\Phi}_{p}, \boldsymbol{\Theta}_{1}, \ldots, \boldsymbol{\Theta}_{q}$ are real or complex $m \times m$ matrices and $\left(\boldsymbol{c}_{k}\right)$ is a sequence of IID $m$-variate zero-mean vectors with non-degenerate covariance matrix $\boldsymbol{\Sigma}_{c}$ characterizing the temporal dependence of its components. There are exisiting works that concern only the autoregressive (AR) modelisation of the spatio-temporal correlations between the clutter [4], [5], [6]. This implies simplifications since the CMLE has a closed form expression in this case. However, the AR model does not necessarily reflect the true correlation structure and it is interesting to study the dual model which is the MA model. The process $\left(\boldsymbol{c}_{k}\right)$ associated to radar clutter returns for example can be non-Gaussian and have for instance a Complex Elliptically Symmetric (CES) distribution [7]. The matrices $\boldsymbol{\Phi}_{i}$ and $\boldsymbol{\Theta}_{i}$ satisfies some constraints to guaranty the existence, uniqueness, causality and invertibility of a solution $\left(\boldsymbol{y}_{k}\right)$ to (1), see e.g. [8]. The estimation of gaussian VARMA models in the space domain by maximizing the likelihood and in the space-frequency domain by maximizing the so called Whittle's approximation to the Gaussian likelihood is considered by [9] and [10], see also [11]. In radar detection schemes, covariance or scatter matrix estimation (for CES distributed clutter) is a fundamental problem. To estimate the scatter matrix of any observed vector under test $\mathbf{y}$ (primary vector on dimension $m$ ), generally it is supposed to dispose of $K>m$ secondary IID vectors (training data) that share with the primary vector the same statistical characteristics [12], [13]. In some applications, as in high spatial resolution radars, the hypothesis of independence (or even of uncorrelation) of the secondary vectors is seldom satisfied due to the nature of the phenomenon at hand. Particularly, for target detection purposes, due to the lack of knowledge of the spectral characteristic of the clutter and the variability of it on long periods of time 


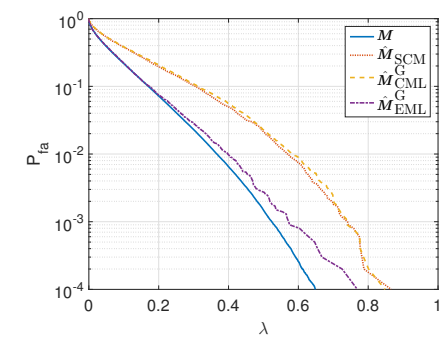

(a)

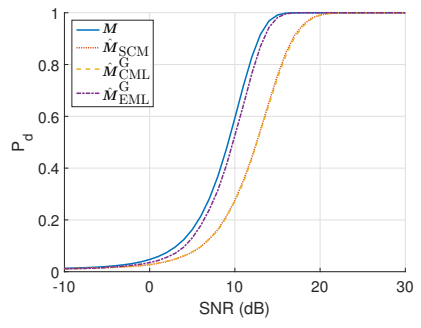

(b)

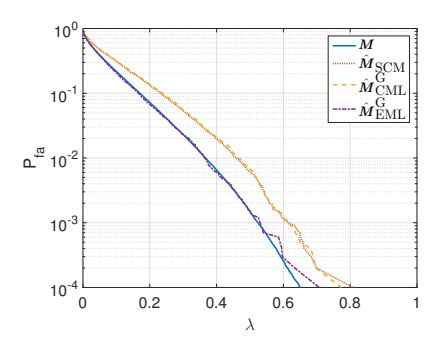

(c)

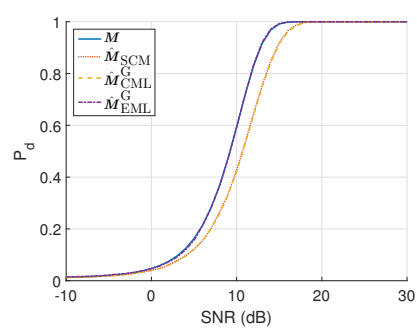

(d)

Fig. 1. $P_{f a}-\lambda$ plot and the corresponding $P_{d}$-SNR relationship for $P_{f a}=10^{-2}$ for spatially non correlated Gaussian clutter $(m=16, \rho=0.5)$. (a), (b) $K=32$. (c), (d) $K=48$.

and large surfaces, the covariance matrix of the clutter must be estimated and plugged into adaptive detectors in both cases of Gaussian (Adaptive Matched Filter, Kelly detector) and non-Gaussian distributed disturbance (Adaptive Normalized Matched Filter). Recent works have also considered spatial correlation [14], [15], [16].

In this paper we assume that the clutter is jointly temporally and spatially correlated. These correlations are modeled through a VARMA process for both Gaussian and non Gaussian signals [17]. Solving this problem can improve the performance of detection. We propose to use the well-known Gaussian Maximum Likelihood (ML) estimator [17] and we extend this formulation for non Gaussian ML formulation in the case of CES innovations. This paper is organized as follows. Section 2 gives a summary of radar detection background and states the problem. Section 3 is dedicated to the estimation of the parameters in the Gaussian case. Section 4 presents the detailed steps of Maximum Likelihood Estimators (MLE) formulation in the non Gaussian case. Section 5 gives some results and discussions about the effect of the spatial and temporal clutter correlations on the radar detection performances through simulated datasets. Conclusions and perspectives are presented in section 6 .

\section{RADAR DETECTION BACKGROUND}

In radar detection theory, the detection problem can be stated as a classical binary hypothesis testing. A received complex clutter observation $\boldsymbol{y}$ is considered as a target-free signal ( $H_{0}$ hypothesis) or a signal containing the target $\left(H_{1}\right.$ hypothesis) as follows,

$$
\left\{\begin{array}{ll}
H_{0}: \boldsymbol{y}=\boldsymbol{c} & \boldsymbol{y}_{k}=\boldsymbol{c}_{k}, k=1, \ldots, K \\
H_{1}: \boldsymbol{y}=\alpha \boldsymbol{p}+\boldsymbol{c} & \boldsymbol{y}_{k}=\boldsymbol{c}_{k}, k=1, \ldots, K
\end{array},\right.
$$

where $\boldsymbol{y}$ is the $m$-vector observed signal, $\boldsymbol{c}_{k}$ is complex secondary data, assumed to be independent, $\alpha$ is the unknown complex target amplitude and $\boldsymbol{p}$ denotes a known steering vector [18]. The goal of the detection theory is to decide between these two hypotheses and the corresponding detection test performance is analyzed through the false alarm probability $P_{f a}$ and the probability of detection $P_{d}$ behaviors. As we propose in this paper to compare performances both in Gaussian and non-Gaussian clutters, we will focus on the well known Normalized Matched Filter (GLRT in partially homogeneous Gaussian clutter [19], approximated GLRT in non-Gaussian CES clutter [20]) defined by

$$
\Lambda_{\mathrm{ANMF}}=\frac{\left|\boldsymbol{p}^{H} \hat{\boldsymbol{M}}^{-1} \boldsymbol{y}\right|^{2}}{\left(\boldsymbol{p}^{H} \hat{\boldsymbol{M}}^{-1} \boldsymbol{p}\right)\left(\boldsymbol{y}^{H} \hat{\boldsymbol{M}}^{-1} \boldsymbol{y}\right)}{\underset{H_{0}}{H_{1}}}_{\gtrless}^{\gtrless},
$$

where $\lambda$ is the detection threshold and where $\hat{M}$ stands for any estimator of the covariance matrix $\boldsymbol{M}$ of $\boldsymbol{y}$. Due to the joint temporal and spatial correlations characterizing the clutter, this estimation is crucial for improving the detection performance. For this reason we attempt in this paper to use a simple adequate time series model that takes into account the spatial correlation of the observed data in order to improve the estimation accuracy. The chosen model is a $\operatorname{VARMA}(0,1)$, i.e. each secondary data is correlated only with the previous secondary data, which leads to the following model where $\boldsymbol{c}_{k}$ is the innovation and $\Theta_{1}$ is a matrix coefficient,

$$
\boldsymbol{y}_{k}=\boldsymbol{c}_{k}+\boldsymbol{\Theta}_{1} \boldsymbol{c}_{k-1}, \quad k=1, \ldots, K .
$$

In the following, we propose to analyze the effect of varying the data distribution structure (independent or spatially correlated) on the radar detection performance under Gaussian and non-Gaussian assumptions. Since the sequence $\left(\boldsymbol{c}_{k}\right)$ is zeromean and IID, the covariance matrix $\boldsymbol{M}=\mathbb{E}\left(\boldsymbol{y}_{k} \boldsymbol{y}_{k}^{H}\right)$ in (4) satisfies

$$
\boldsymbol{M}=\mathbb{E}\left(\boldsymbol{c}_{k} \boldsymbol{c}_{k}^{H}\right)+\boldsymbol{\Theta}_{1} \mathbb{E}\left(\boldsymbol{c}_{k} \boldsymbol{c}_{k}^{H}\right) \boldsymbol{\Theta}_{1}^{H}=\boldsymbol{\Sigma}_{c}+\boldsymbol{\Theta}_{1} \boldsymbol{\Sigma}_{c} \boldsymbol{\Theta}_{1}^{H} .
$$

\section{GAUSSIAN CASE}

Here, we assume that the observations $\boldsymbol{y}_{k}$ follow a Gaussian distribution and we analyze the two cases where the data are either independent or follow a $\operatorname{VARMA}(0,1)$ model.

\section{A. Independent observations}

For the rest of this paper, let $\boldsymbol{Y}=\left(\boldsymbol{y}_{1}^{T}, \boldsymbol{y}_{2}^{T}, \ldots, \boldsymbol{y}_{K}^{T}\right)^{T}$. For independent Gaussian distributed data, the likelihood function $L_{1}(\boldsymbol{Y}, \boldsymbol{M})$ is given by

$$
L_{1}(\boldsymbol{Y} ; \boldsymbol{M})=\frac{1}{\pi^{m K}|\boldsymbol{M}|^{K}} \exp \left(-\sum_{k=1}^{K} \boldsymbol{y}_{k}^{H} \boldsymbol{M}^{-1} \boldsymbol{y}_{k}\right),
$$




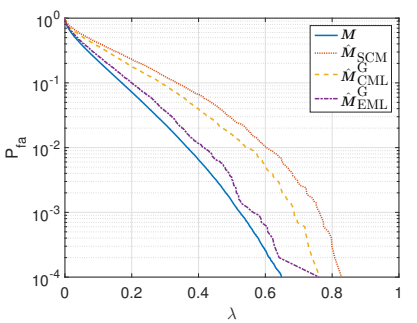

(a)

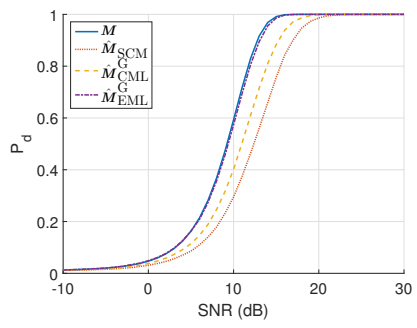

(b)

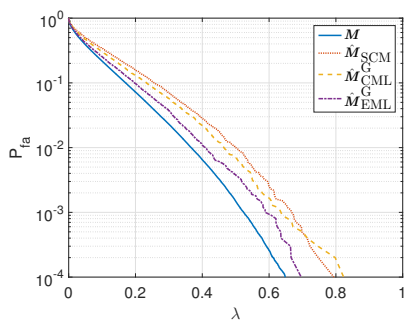

(c)

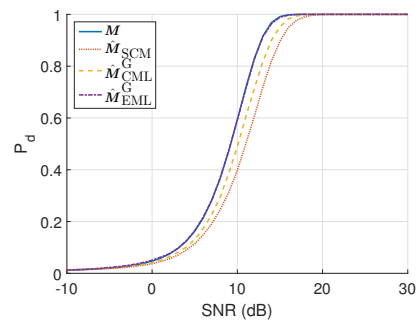

(d)

Fig. 2. $P_{f a}-\lambda$ plot and the corresponding $P_{d}$-SNR relationship for $P_{f a}=10^{-2}$ for spatially VARMA( 0,1$)$ correlated Gaussian clutter $(m=16, \rho=0.5$, $\Theta_{1}=0.9 \boldsymbol{I}_{\boldsymbol{m}}$ ). (a), (b) $K=32$. (c), (d) $K=48$.

where $|\boldsymbol{M}|=\operatorname{det}(\boldsymbol{M})$. The maximum of $L_{1}(\boldsymbol{Y} ; \boldsymbol{M})$ with respect to $M$ is the well-known Sample Covariance Matrix (SCM) $\hat{M}_{\mathrm{SCM}}$ given by

$$
\hat{\mathbf{M}}_{\mathrm{SCM}}=\frac{1}{K} \sum_{k=1}^{K} \boldsymbol{y}_{k} \boldsymbol{y}_{k}^{H} .
$$

\section{B. $\operatorname{VARMA}(0,1)$ observations}

The VARMA $(0,1)$ model (4) is specified by the covariance matrix $\boldsymbol{\Sigma}_{c}$ of the innovations $\boldsymbol{c}_{k}$ and the coefficients matrix $\Theta_{1}$. The estimation techniques of these parameters are based on the conditional or the exact MLE [17].

1) Conditional likelihood function: The conditional MLE is obtained by assuming that $\boldsymbol{c}_{0}=\mathbf{0}$. It follows from (4) that $\boldsymbol{C}=\left(\boldsymbol{c}_{1}^{T}, \boldsymbol{c}_{2}^{T}, \ldots, \boldsymbol{c}_{K}^{T}\right)^{T}$ is related to $\boldsymbol{Y}$ by $\boldsymbol{C}=\boldsymbol{T} \boldsymbol{Y}$ where

$$
\boldsymbol{T}=\left[\begin{array}{cccc}
\boldsymbol{I}_{m} & \mathbf{0}_{m} & \ldots & \mathbf{0}_{m} \\
-\boldsymbol{\Theta}_{1} & \boldsymbol{I}_{m} & \ldots & \mathbf{0}_{m} \\
\left(-\boldsymbol{\Theta}_{1}\right)^{2} & -\boldsymbol{\Theta}_{1} & \ldots & \mathbf{0}_{m} \\
\vdots & \vdots & \ddots & \vdots \\
\left(-\boldsymbol{\Theta}_{1}\right)^{K-1} & \left(-\boldsymbol{\Theta}_{1}\right)^{K-2} & \ldots & \boldsymbol{I}_{m}
\end{array}\right]
$$

Since the innovations $\boldsymbol{c}_{k}$ are IID and $\operatorname{det}(\boldsymbol{T})=1$, the conditional likelihood function of $\boldsymbol{Y}$ is

$$
L_{2}\left(\boldsymbol{Y} ; \boldsymbol{\Theta}_{1}, \boldsymbol{\Sigma}_{\boldsymbol{c}}\right)=\frac{1}{\pi^{m K}\left|\boldsymbol{\Sigma}_{c}\right|^{K}} \exp \left(-\sum_{k=1}^{K} \boldsymbol{c}_{k}^{H} \boldsymbol{\Sigma}_{c}^{-1} \boldsymbol{c}_{k}\right) \text {. }
$$

We denote by $\hat{\boldsymbol{\Theta}}_{1, \mathrm{CML}}^{\mathrm{G}}$ and $\hat{\boldsymbol{\Sigma}}_{c, \mathrm{CML}}^{\mathrm{G}}$ the values of $\boldsymbol{\Theta}_{1}$ and $\boldsymbol{\Sigma}_{\boldsymbol{c}}$ which maximize (9), and we denote by $\hat{\boldsymbol{M}}_{\mathrm{CML}}^{\mathrm{G}}$ the value of $\boldsymbol{M}$ obtained by replacing respectively $\boldsymbol{\Theta}_{1}$ and $\boldsymbol{\Sigma}_{c}$ by $\hat{\boldsymbol{\Theta}}_{1, \mathrm{CML}}^{\mathrm{G}}$ and $\hat{\boldsymbol{\Sigma}}_{c, \mathrm{CML}}^{\mathrm{G}}$ in (5).

2) Exact likelihood function: The different steps in computing the exact likelihood function are similar to the ones described in the conditional case except that we must estimate the value of the initial innovation $\boldsymbol{c}_{0}$. Similarly to the conditional case, we observe that the determinant of the matrix relating the observations to the innovations is equal to one, and then the joint Probability Density Function (PDF) of the observations $\boldsymbol{y}_{k}$ is equal to the product of the PDF's of the innovations, i.e.,

$$
p\left(\boldsymbol{c}_{0}, \boldsymbol{y}_{1}, \boldsymbol{y}_{2}, \ldots, \boldsymbol{y}_{K}\right)=\prod_{k=0}^{K} p\left(\boldsymbol{c}_{k}\right)
$$

The exact likelihood function $L_{3}\left(\boldsymbol{Y} ; \boldsymbol{\Theta}_{1}, \boldsymbol{\Sigma}_{c}\right)$ is obtained by integrating (10) out $\boldsymbol{c}_{0}$. It was shown in [17] that

$$
L_{3}\left(\boldsymbol{Y} ; \boldsymbol{\Theta}_{1}, \boldsymbol{\Sigma}_{\boldsymbol{c}}\right)=\frac{\exp \left(-\left(\tilde{\boldsymbol{Y}}-\tilde{\boldsymbol{X}} \hat{\boldsymbol{c}}_{0}\right)^{H}\left(\tilde{\boldsymbol{Y}}-\tilde{\boldsymbol{X}} \hat{\boldsymbol{c}}_{0}\right)\right)}{\pi\left|\boldsymbol{\Sigma}_{c}\right|^{K}\left|\tilde{\boldsymbol{X}}^{H} \tilde{\boldsymbol{X}}\right|},
$$

where $\boldsymbol{c}_{0}, \tilde{\boldsymbol{X}}$ and $\tilde{\boldsymbol{Y}}$ depend only on the observations and $\boldsymbol{\Sigma}_{c}$ and $\boldsymbol{\Theta}_{1}$. We denote by $\hat{\boldsymbol{\Theta}}_{1, \mathrm{EML}}^{\mathrm{G}}$ and $\hat{\boldsymbol{\Sigma}}_{c \text {,EML }}^{\mathrm{G}}$ the values of $\boldsymbol{\Theta}_{1}$ and $\boldsymbol{\Sigma}_{c}$ that maximize (11). From these estimated matrices we compute $\hat{M}_{\mathrm{EML}}^{\mathrm{G}}$ using (5). The exact procedure is particularly computationally expensive to solve when $\boldsymbol{\Sigma}_{c}$ is not structured $(m \times(m+1) / 2$ elements to estimate). In this paper, we have solved the problem when $\boldsymbol{\Sigma}_{c}$ has a Toeplitz structure depending on only one parameter.

As the sample size tends to infinity, the conditional MLE's $\hat{\boldsymbol{\Theta}}_{1, \mathrm{CML}}^{\mathrm{G}}$ and $\hat{\boldsymbol{\Sigma}}_{c, \mathrm{CML}}^{\mathrm{G}}$, and the exact MLE's $\hat{\boldsymbol{\Theta}}_{1, \mathrm{EML}}^{\mathrm{G}}$ and $\hat{\boldsymbol{\Sigma}}_{c, \mathrm{EML}}^{\mathrm{G}}$ both converge almost surely to the true values $\boldsymbol{\Theta}_{1}$ and $\boldsymbol{\Sigma}_{c}$. But their finite sample behaviours may be different, especially in the case of small datasets.

\section{Non Gaussian CASE}

In real measurements, the clutter is often non-Gaussian distributed. One of the main class of these non Gaussian statistical models is the compound Gaussian processes (CES subclass). The random vector $\boldsymbol{c}_{k}$ is compound Gaussian if $\boldsymbol{c}_{k}=\sqrt{\tau_{k}} \boldsymbol{x}_{k}$ where $\tau_{k}$ is a positive random variable called texture which measures the variability of the local power of the received signal, and $\boldsymbol{x}_{k}$ is a $m$-dimensional zero-mean Gaussian vectors with covariance matrix $\boldsymbol{\Sigma}_{x}$ called speckle, and $\tau_{k}$ and $\boldsymbol{x}_{k}$ are independent. We assume in the following that the clutter texture is Gamma distributed. Since the texture and the speckle are independent, the covariance matrix of the data $\boldsymbol{c}_{k}$ is simply given by $\boldsymbol{\Sigma}_{c}=\mathbb{E}\left(\tau_{k}\right) \boldsymbol{\Sigma}_{x}$. For identifiability, we assume that $\mathbb{E}\left(\tau_{k}\right)=1$. In the following, we investigate the case where the data are either independent or follow a $\operatorname{VARMA}(0,1)$ model.

\section{A. Independent observations}

In this case, $\boldsymbol{y}_{k}=\boldsymbol{c}_{k}=\sqrt{\tau_{k}} \boldsymbol{x}_{k}$ which gives $\boldsymbol{M}=\boldsymbol{\Sigma}_{c}$. The conditional PDF of $\boldsymbol{y}_{k}$ conditionally to $\tau_{k}$ is

$$
p\left(\boldsymbol{y}_{k} \mid \tau_{k}\right)=\frac{1}{\pi^{m}|\boldsymbol{M}| \tau_{k}^{m}} \exp \left(-\frac{\boldsymbol{y}_{k}^{H} \boldsymbol{M}^{-1} \boldsymbol{y}_{k}}{\tau_{k}}\right) .
$$




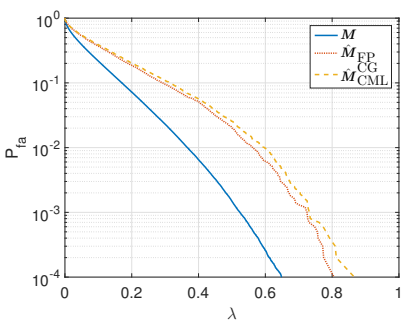

(a)

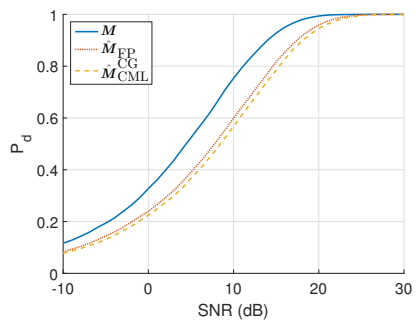

(b)

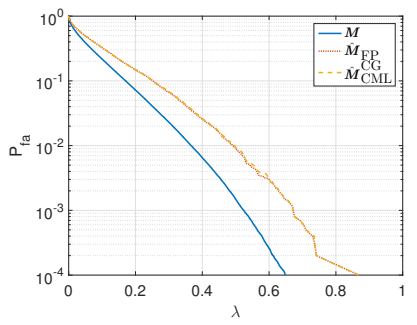

(c)

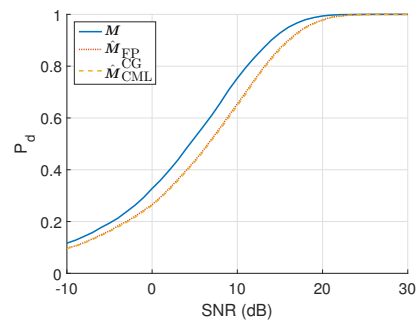

(d)

Fig. 3. $P_{f a}-\lambda$ plot and the corresponding $P_{d}$-SNR relationship for $P_{f a}=10^{-2}$ for spatially non correlated compound Gaussian clutter $(\nu=0.5, m=16$, $\rho=0.5$ ). (a), (b) $K=32$. (c), (d) $K=48$.

The value of $\tau_{k}$ that maximizes $p\left(\boldsymbol{y}_{k} \mid \tau_{k}\right)$ is $\hat{\tau}_{k}=$ $\boldsymbol{c}_{k}^{H} \boldsymbol{\Sigma}_{c}^{-1} \boldsymbol{c}_{k} / m=\boldsymbol{y}_{k}^{H} \boldsymbol{M}^{-1} \boldsymbol{y}_{k} / m$, see e.g. [21]. Replacing $\tau_{k}$ by $\hat{\tau}_{k}$ in (12), we get

$$
L_{4}(\boldsymbol{Y} ; \boldsymbol{M})=\prod_{k=1}^{K} \frac{m^{m} \exp (-m)}{\pi^{m}|\boldsymbol{M}|\left(\boldsymbol{y}_{k}^{H} \boldsymbol{M}^{-1} \boldsymbol{y}_{k}\right)^{m}} .
$$

The partial derivative of (13) with respect to $M$ leads to the Tyler's estimator [22] which is defined as the unique solution of the following fixed point equation

$$
\hat{\boldsymbol{M}}_{\mathrm{FP}}=\frac{m}{K} \sum_{k=1}^{K} \frac{\boldsymbol{y}_{k} \boldsymbol{y}_{k}^{H}}{\boldsymbol{y}_{k}^{H} \hat{\boldsymbol{M}}_{\mathrm{FP}}^{-1} \boldsymbol{y}_{k}},
$$

and it can be noticed that this estimate does not depend on the texture.

\section{B. $\operatorname{VARMA}(0,1)$ observations}

We assume that $\boldsymbol{y}_{k}$ satisfies (4) where $\boldsymbol{c}_{k}$ is compound Gaussian, and we investigate the conditional MLE's of $\boldsymbol{\Theta}_{1}$ and $\boldsymbol{\Sigma}_{c}$ obtained by assuming that $\boldsymbol{c}_{0}=\mathbf{0}$. The conditional PDF of $\boldsymbol{c}_{k}$ conditionally to $\tau_{k}, p\left(\boldsymbol{c}_{k} \mid \tau_{k}\right)$ is given by (12) where $\boldsymbol{y}_{k}$ is replaced by $\boldsymbol{c}_{k}$. Replacing $\tau_{k}$ by $\hat{\tau}_{k}=\boldsymbol{c}_{k}^{H} \boldsymbol{\Sigma}_{c}^{-1} \boldsymbol{c}_{k} / m$ in $p\left(\boldsymbol{c}_{k} \mid \tau_{k}\right)$ and using the same arguments as in Section III-B1, we obtain the conditional likelihood function of $\boldsymbol{Y}$,

$$
L_{5}\left(\boldsymbol{Y} ; \boldsymbol{\Theta}_{1}, \boldsymbol{\Sigma}_{\boldsymbol{c}}\right)=\prod_{k=1}^{K} \frac{m^{m} \exp (-m)}{\pi^{m}\left|\boldsymbol{\Sigma}_{c}\right|\left(\boldsymbol{c}_{k}^{H} \boldsymbol{\Sigma}_{c}^{-1} \boldsymbol{c}_{k}\right)^{m}} .
$$

Since $\boldsymbol{C}=\boldsymbol{T} \boldsymbol{Y}$ where $\boldsymbol{T}$ is given by (8) and involves only $\Theta_{1}$, the maximum of (15) with respect to $\boldsymbol{\Sigma}_{c}$ is $\hat{\boldsymbol{\Sigma}}_{c, \mathrm{FP}}$ given by (14) where $\hat{\boldsymbol{M}}_{\mathrm{FP}}$ is replaced by $\hat{\boldsymbol{\Sigma}}_{c, \mathrm{FP}}$. Then, replacing $\boldsymbol{\Sigma}_{c}$ by $\hat{\boldsymbol{\Sigma}}_{c, \mathrm{FP}}$ in (15) and maximizing with respect to $\boldsymbol{\Theta}_{1}$, we obtain $\hat{\boldsymbol{\Theta}}_{1, \mathrm{CML}}^{\mathrm{CG}}$. Replacing respectively $\boldsymbol{\Theta}_{1}$ and $\boldsymbol{\Sigma}_{c}$ by $\hat{\boldsymbol{\Theta}}_{1, \mathrm{CML}}^{\mathrm{CG}}$ and $\hat{\boldsymbol{\Sigma}}_{c, \mathrm{FP}}$ in (5), we obtain $\hat{\boldsymbol{M}}_{\mathrm{CML}}^{\mathrm{CG}}$.

\section{RESULTS AND DISCUSSIONS}

We have evaluated here both the conditional/exact likelihood functions in Gaussian and in non-Gaussian cases for simulated independent or correlated data. All the results are given with $m=16$ and $K=32$ and 48 respectively and they are presented with $n=10^{4}$ Monte Carlo measurements. In the case of Gaussian assumption, we maximize the likelihood functions $L_{1}, L_{2}$ and $L_{3}$ with respect to $\boldsymbol{\Sigma}_{c}$ and $\boldsymbol{\Theta}_{1}$ simultaneously. Whereas in the case of non Gaussian estimations, only the maximization with respect to $\Theta_{1}$ is performed since the optimal covariance matrix $\hat{\boldsymbol{\Sigma}}_{c}$ is reached with the fixed point algorithm. For the exact likelihood function, the optimization is initialized with initial values $\boldsymbol{\Theta}_{1,0}$ and $\boldsymbol{\Sigma}_{c, 0}$ obtained from the Yule Walker estimator after transforming the $\operatorname{VARMA}(0,1)$ model to a Auto Regressive (AR) one [17]. As previously discussed in section III in order to reduce calculation complexity, we assume here that the matrix $\Theta_{1}$ is a diagonal one and that the innovation covariance matrix $\boldsymbol{\Sigma}_{c}$ has a simple Toeplitz structure $\left(\boldsymbol{\Sigma}_{c}\right)_{i, j}=\rho^{|i-j|}$ for $i, j \in[1, m]$ with $\rho=0.5$.

\section{A. Estimation in Gaussian environment}

The performance of ANMF (3) is evaluated in spatially uncorrelated and correlated Gaussian environments for four covariance matrix estimates: the benchmark ( $M$ is known), the SCM $\hat{M}_{\mathrm{SCM}}$, the $\operatorname{VARMA}(0,1)$ conditional likelihood estimate $\hat{\boldsymbol{M}}_{\mathrm{CML}}$ and the $\operatorname{VARMA}(0,1)$ exact one $\hat{\boldsymbol{M}}_{\mathrm{EML}}$. For each detector, we plot the relationship between $P_{f a}$ and the detection threshold $\lambda$. Defining the Signal to Noise Ratio (SNR) as $\alpha^{2} \boldsymbol{p}^{H} \boldsymbol{M}^{-1} \boldsymbol{p}$, these detectors are evaluated in terms of $P_{d}$-SNR performance for $P_{f a}=10^{-2}$. Figure 1 shows the corresponding results for $K=32$ and $K=48$. In this figure we can notice that the exact likelihood function calculated for $\operatorname{VARMA}(0,1)$ data gives best detection performance (except for benchmark). This is explained by the fact that since the covariance matrix $\boldsymbol{\Sigma}_{c}$ of the innovations has a specific Toeplitz structure, we maximize the exact ML function with respect to the factor $\rho$ (only one parameter to estimate). This choice allow to speed-up the maximization task due to the simple Toeplitz structure of the estimated $\hat{\boldsymbol{\Sigma}}_{c}$. The performance related to the conditional likelihood tends here to have the same performance as SCM. The figure 2 shows the case of spatially correlated $\operatorname{VARMA}(0,1)$ data for $\boldsymbol{\Theta}_{1}=0.9 \boldsymbol{I}_{m}$. In this figure, the conditional likelihood detector has better performance than the SCM one. This is explained by the fact that the SCM estimator does not take into account the spatial correlation of the clutter. Again, we can notice that the exact likelihood estimator gives the nearest results to the optimal benchmarks obtained by the theoretical covariance matrix $M$ but this is always due to the optimization constraint we have imposed to the particular Toeplitz matrix. 


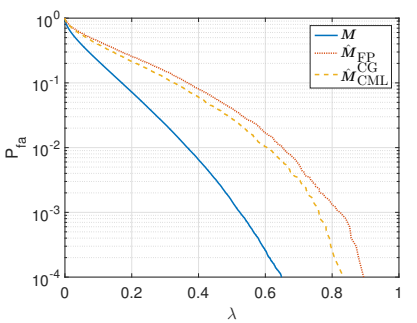

(a)

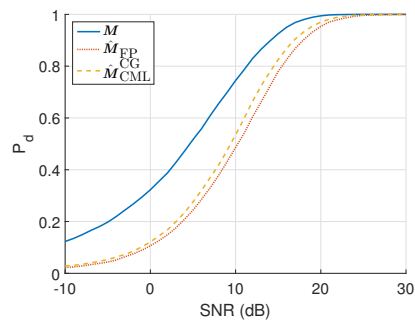

(b)

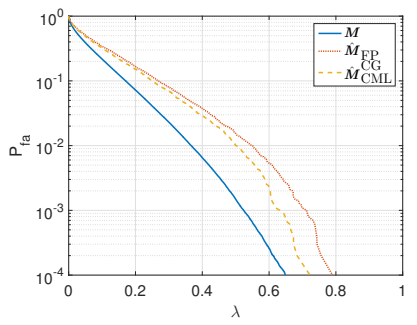

(c)

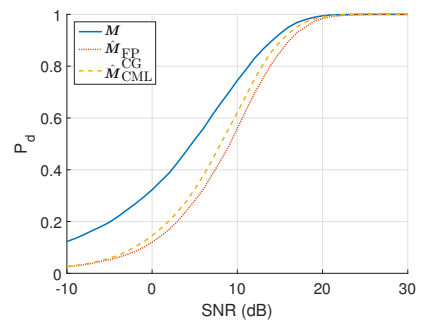

(d)

Fig. 4. $P_{f a}-\lambda$ plot and the corresponding $P_{d}$-SNR relationship for $P_{f a}=10^{-2}$ for VARMA $(0,1)$ spatially correlated compound Gaussian clutter $(\nu=0.5$, $m=16, \rho=0.5, \boldsymbol{\Theta}_{\mathbf{1}}=0.9 \boldsymbol{I}_{\boldsymbol{m}}$ ). (a), (b) $K=32$. (c), (d) $K=48$.

\section{B. Estimation in compound Gaussian environment}

The performance of ANMF (3) is evaluated in spatially uncorrelated and correlated compound Gaussian data environment. Instead of the SCM estimate, we use here the Tyler's estimator of the innovations covariance matrix as given in (14). The figure 3 shows the $P_{f a}$ and the $P_{d}$ values for both Tyler's and conditional ML estimate of the covariance matrix for $K=32$ and $K=48$. We can observe that the Tyler's estimator is a little bit better than the conditional ML one for small number of observations $(K=32)$. The second dataset have been generated under highly spatially correlated $\operatorname{VARMA}(0,1)$ model with $\boldsymbol{\Theta}_{1}=0.9 \boldsymbol{I}_{m}$. As in the case of the Gaussian data, the spatial correlation characterizing the clutter disrupts the accuracy of the Tyler's estimator and the conditional ML gives slight better performance as illustrated in figure 4.

\section{CONCLUSION}

In this paper, we proved that the spatial correlation characterizing the clutter secondary data could be exploited to enhance the performance of detection. This enhancement takes place in simulated Gaussian distributed data through a maximization of both conditional and exact likelihood functions to estimate the data covariance matrix. In the case of non Gaussian distributed clutter, the conditional ML gives a considerable improvement of the quality of detection with respect to the classical Tyler's estimator. The success of this estimator will motivate us to test it on real radar datasets and to estimate the covariance matrix by calculating the non-Gaussian exact likelihood function.

\section{REFERENCES}

[1] T. S. Rao and A. M. C. Antunes, "Spatio-temporal modelling of temperature time series: a comparative study," in Time series analysis and applications to geophysical systems, pp. 123-150, Springer, 2004.

[2] A. Cliff and J. K. Ord, "Space-time modelling with an application to regional forecasting," Transactions of the Institute of British Geographers, pp. 119-128, 1975.

[3] M. Greco, F. Gini, and M. Rangaswamy, "Statistical analysis of measured polarimetric clutter data at different range resolutions," IEE Proceedings-Radar, Sonar and Navigation, vol. 153, no. 6, pp. 473481, 2006.

[4] A. L. Swindlehurst and P. Stoica, "Maximum likelihood methods in radar array signal processing," Proceedings of the IEEE, vol. 86, no. 2, pp. 421-441, 1998.
[5] J. R. Roman, M. Rangaswamy, D. W. Davis, Q. Zhang, B. Himed, and J. H. Michels, "Parametric adaptive matched filter for airborne radar applications," IEEE Transactions on Aerospace and Electronic Systems, vol. 36, no. 2, pp. 677-692, 2000.

[6] K. J. Sohn, H. Li, and B. Himed, "Parametric glrt for multichannel adaptive signal detection," in Sensor Array and Multichannel Processing, 2006. Fourth IEEE Workshop on, pp. 399-403, IEEE, 2006.

[7] E. Ollila, D. E. Tyler, V. Koivunen, and H. V. Poor, "Complex elliptically symmetric distributions: Survey, new results and applications," Signal Processing, IEEE Transactions on, vol. 60, pp. 5597 -5625, nov. 2012.

[8] P. J. Brockwell and R. A. Davis, Time series: theory and methods. Springer Science \& Business Media, 2013.

[9] M. Deistler, W. Dunsmuir, and E. Hannan, "Vector linear time series models: corrections and extensions," Advances in Applied Probability, vol. 10 , no. 02 , pp. $360-372,1978$.

[10] W. Dunsmuir, "A central limit theorem for parameter estimation in stationary vector time series and its application to models for a signal observed with noise," The Annals of Statistics, pp. 490-506, 1979.

[11] G. C. Reinsel, Elements of multivariate time series analysis. Springer Science \& Business Media, 2003.

[12] E. J. Kelly, "An adaptive detection algorithm," IEEE transactions on aerospace and electronic systems, no. 2, pp. 115-127, 1986.

[13] S. S. Haykin and A. O. Steinhardt, Adaptive radar detection and estimation, vol. 11. Wiley-Interscience, 1992.

[14] A. Aubry, A. De Maio, G. Foglia, C. Hao, and D. Orlando, "Radar detection and range estimation using oversampled data," IEEE Transactions on Aerospace and Electronic Systems, vol. 51, no. 2, pp. 1039-1052, 2015.

[15] C. Hao, D. Orlando, G. Foglia, X. Ma, and C. Hou, "Adaptive radar detection and range estimation with oversampled data for partially homogeneous environment," IEEE Signal Processing Letters, vol. 22, no. 9, pp. 1359-1363, 2015.

[16] R. Couillet, M. S. Greco, J.-P. Ovarlez, and F. Pascal, "Rmt for whitening space correlation and applications to radar detection," in Computational Advances in Multi-Sensor Adaptive Processing (CAMSAP), 2015 IEEE 6th International Workshop on, pp. 149-152, IEEE, 2015.

[17] R. S. Tsay, Multivariate Time Series Analysis: with $R$ and financial applications. John Wiley \& Sons, 2013.

[18] J.-P. Ovarlez, F. Pascal, and P. Forster, "Covariance matrix estimation in sirv and elliptical processes and their applications in radar detection," 2015.

[19] S. Kraut and L. L. Scharf, "The cfar adaptive subspace detector is a scale-invariant glrt," IEEE Transactions on Signal Processing, vol. 47, no. 9, pp. 2538-2541, 1999.

[20] M. Rangaswamy, D. D. Weiner, and A. Ozturk, "Non-gaussian random vector identification using spherically invariant random processes," IEEE Transactions on Aerospace and Electronic Systems, vol. 29, no. 1, pp. 111-124, 1993.

[21] F. Gini and M. Greco, "Covariance matrix estimation for CFAR detection in correlated heavy tailed clutter," Signal Processing, vol. 82, no. 12, pp. 1847-1859, 2002.

[22] D. Tyler, "A distribution-free $M$-estimator of multivariate scatter," The Annals of Statistics, vol. 15, no. 1, pp. 234-251, 1987. 